\title{
PODER, LIDERAZGO Y CONTROL DE LAS DISTRIBUIDORAS EN LA HORTICULTURA DE EXPORTACIÓN SINALOENSE
}

\section{POWER, LEADERSHIP AND CONTROL OF THE DISTRIBUTION COMPANIES IN THE EXPORT OF FRESH VEGETABLES FROM SINALOA, MEXICO}

\section{Ezequiel Avilés-Ochoa1 y Lydia María López-Barraza2}

Profesor Investigador de la Universidad de Occidente, pertenece al Sistema Nacional de Investigadores Nivel II, correo electrónico: eaviles@culiacan.udo.mx1. Coordinadora de la Maestría en Administración de la Universidad de Occidente, Unidad Culiacán, pertenece al Sistema Sinaloense de Investigadores y Tecnólogos del Consejo Estatal de Ciencia y Tecnología, correo electrónico: lbarraza@culiacan.udo.mx2.

\section{RESUMEN}

Un término clave para la construcción del paradigma de cadenas globales de valor es el de gobernanza, concepto en el cual las variables poder, liderazgo y control son fundamentales. La investigación se enfoca en las distribuidoras de hortalizas sinaloenses en el mercado norteamericano, ya que permiten descubrir la interacción entre regiones de distintos países para la creación de una cadena hortícola de valor. El resultado es un índice que define cuál de los actores, que participa en la comercialización de vegetales frescos, ejerce mayor gobernanza.

Palabras clave: gobernanza, comercio hortícola, distribución, cadenas de supermercados, cadena hortícola de valor, Sinaloa.

\section{SUMMARY}

A key concept in the construction of the paradigm of global value chains is governance, for its constitution the variables of power, leadership and control are essential. The research focuses on Sinaloa vegetable distributors in the U.S.A. market, which reveals the interaction between regions of different countries for the formation of a horticultural value chain. The result is an index that defines which of the

Recibido: 04 de diciembre de 2011. Aceptado: 02 de marzo de 2012. Publicado como ARTÍCULO CIENTÍFICO en Ra Ximhai 8(3): 233-246. actors, involved in the fresh produce trade, practices more governance.

Keywords: governance, fresh produce trade, distribution, supermarket chains, horticultural value chains, Sinaloa.

\section{INTRODUCCIÓN}

Un término clave en la construcción del paradigma de Cadenas Globales de Valor es el de gobernanza. Sin gobernanza las cadenas no pueden existir. Para la formación de este concepto las variables de poder, liderazgo $\mathrm{y}$ control son fundamentales, por ello su estudio es clave para su entendimiento e impacto en las cadenas de valor.

La investigación se enfoca en las distribuidoras de hortalizas sinaloenses en el mercado norteamericano de invierno, instaladas en Nogales Arizona y examina la interacción entre regiones de distintos países, tal es el caso de México y Estados Unidos para la formación de una cadena hortícola de valor. Al mismo tiempo, proporcionan la comprensión de nuevas realidades en materia de mercado.

El resultado es la construcción de un índice que mide el impacto de las variables de poder, liderazgo y control, con el fin de definir

Los actores son productor, distribuidor, cadena de supermercado y centrales de abasto.

Sinaloa junto con Florida, durante los meses de invierno, aportan 80\% del abasto en Estados Unidos. 
desde la perspectiva de las distribuidoras, cuál de los actores que participa en la comercialización hortícola, ejerce mayor gobernanza en la cadena y por lo tanto, establecen y monitorean los parámetros que el resto deben cumplir.

La Cadena Hortícola de Valor SinaloaEstados Unidos (CHVSEU)

Desde fines del siglo XIX, Sinaloa, estado federado de México, ya envía sus primeras exportaciones al país vecino del norte. A partir de la década de los treinta, la entidad adopta un modelo de desarrollo primario-exportador. Ello fue resultado, principalmente, de la construcción de grandes obras hidráulicas, caminos y carreteras en la entidad. Aunado a eso, la conexión vía ferrocarril Sud Pacífico facilitó la comercialización de productos agrícolas con la Unión Americana. De esa forma, la agricultura orientada a la exportación se convierte en una de las actividades motoras de la economía sinaloense.

Este proceso ocurrió en un escenario favorable, marcado por el aumento del ingreso per cápita en los Estados Unidos de América (EE.UU.) y el embargo comercial a Cuba; también, por las ventajas naturales en la producción de otoño-invierno y la capacidad de respuesta de los productores sinaloenses.

En 1962, el gobierno norteamericano decreta un embargo comercial a Cuba, hasta entonces el principal proveedor de hortalizas frescas al mercado norteamericano (VanSickle, et al., 1994: 1). Esta decisión de política comercial, permite crear el espacio para que México, Sinaloa de manera

Para tal efecto, el TLCAN establece cuotas y periodos largos de desgravación, salvaguardas especiales, trato especial y la exclusión en ciertos productos. En esta liberalización regulada, los Estados Unidos lograron una excelente negociación: conservar protegido sus intereses en cítricos, frutas y hortalizas. fundamental, se convierta en el principal abastecedor de la demanda estadounidense en el mercado de invierno. En los setenta, el sector hortícola de exportación mexicano ya tiene un significativo posicionamiento. Para 1994, cuando entra en operación el Tratado de Libre Comercio de América del Norte (TLCAN), suscrito con EE.UU. y Canadá, la comercialización de vegetales en fresco ya está consolidada.

La región de Nogales, Arizona, distante a poco más de 1,100 kilómetros de Sinaloa, ha estado vinculada históricamente a la exportación hortícola mexicana; ubicada en la frontera que separa a México de EE.UU., se ha caracterizado por ser el punto de venta de los productos hortícolas de exportación provenientes de Sinaloa; fue fundada en 1880 por Jacob Isaacson, y dos años después, se convierte en un sitio de interconexión del ferrocarril entre México y Estados Unidos, haciendo del intercambio comercial el impulsor de su dinámica productiva.

La comercialización hortícola en la frontera inicia, después de 1908, con la entrada en vigor del servicio de ferrocarril hacia Tucson. La actividad tiene un giro importante a principios de los años 50, con el uso de los carros refrigerados que permitían la descarga y redistribución del producto, haciendo posible la penetración de los vegetales frescos a un gran número de ciudades estadounidenses. De esa forma, la frontera de Nogales, se posiciona como una región estratégica en la distribución hortícola, al servir de puente de los productores sinaloenses con el mercado estadounidense. Ello conduce a la especialización geográfica en el grupo de actividades relacionadas con

\footnotetext{
Descripción de la gama completa de actividades que requiere un producto en sus diversas fases de producción hasta su entrega al consumidor final y de su uso posterior.

El término gobernanza, como concepto central en la CGV, es usado para expresar el desempeño de algunas firmas que establecen y hacen cumplir los parámetros bajo los cuales otras deben manejarse.
} 
la horticultura.

Mientras en Sinaloa se producen y empacan las hortalizas, en Nogales se lleva a cabo la etapa de distribución al intermediario y al consumidor final, ya sean éstos individuos u organizaciones.

Las características de la actividad hortícola de exportación sinaloense, y su relación con la región de Nogales y diversos mercados estadounidenses, hacen posible estudiar la correspondencia comercial en el contexto de una Cadena Hortícola de Valor (CHV), integrada por la región de Sinaloa y los Estados Unidos (CHVSEU).

Para analizar la formación de la $\mathrm{CHV}$ es necesario hacer uso del paradigma de Cadenas Globales de Valor (CGV) y de su concepto central: la gobernanza. Esta teoría integra a un conjunto de paradigmas afines a los temas de localización, encadenamientos, crecimiento económico, interacción espacial, distritos industriales y el modelo de las ventajas competitivas de Michael Porter (1980).

Este marco referencial, permite entender y explicar la inserción de una región orientada a la producción de exportación en un esquema internacional de mercado. También posibilita abordar el liderazgo de una firma, o un grupo de ellas, a lo largo del encadenamiento, estableciendo cierto orden en los nodos, representado por las acciones desplegadas por las empresas distribuidoras. Bajo ese ordenamiento, es posible analizar si las distribuidoras hortícolas, ubicadas en el suroeste de EE.UU., ejercen su gobernanza en la cadena.

\footnotetext{
La gobernanza jerárquica funciona de la siguiente manera: las cadenas indican a la distribuidora el tipo de producto, cantidad y calidad que necesitan, además de solicitar presentaciones y productos especiales, de acuerdo a las exigencias del consumidor; por su parte, la distribuidora atiende las demandas de las cadenas al transmitir al productor los gustos y preferencias del cliente, y certifica el cumplimiento de los requerimientos.
}

La teoría postula que las empresas que comercializan de manera internacional desempeñanelliderazgodelencadenamiento, estableciendo y monitoreando los parámetros que otras deben cumplir. Así, las distribuidoras hortícolas son sujetas y a su vez ejercen gobernanza, que permite no sólo la modelación de relaciones de mercado en el comercio entre México y EE.UU. sino la formación de una cadena hortícola de valor, resultado del encadenamiento de diversas firmas interdependientes.

La gobernanza jerárquica, prevaleciente en la CHVSEU, es ejercida por las cadenas de supermercados sobre las distribuidoras $\mathrm{y}$, en un segundo plano, de éstas a los productores. Las distribuidoras la expresan a través del conocimiento de las normas de calidad, la vanguardia en innovación tecnológica, la permanencia de sistemas informativos, la participación en el diseño de políticas públicas, así como en el empleo y puesta en práctica de las variables: poder, liderazgo y control. Todas estas acciones se desempeñan en un ambiente de confianza, el cual garantiza relaciones comerciales y sociales duraderas.

En la gobernanza de las distribuidoras destaca la importancia de la calidad del producto, parte relevante en la determinación de la competencia y permanencia en el mercado. Las distribuidoras expresan las pautas de producción y comercialización, y son importantes para la determinación de los volúmenes de producción, diversificación de productos y la formación de precios; también, en el establecimiento de los estándares y normas, al ser vías de transmisión de las 
señales de mercado y de regulaciones.

El Papel de la Gobernanza: ¿Quién lo juega? El abordaje de la CGV está centrado en la gama de actores que ejercen poder al interior de los sistemas de producción y distribución. En este tipo de agrupamiento, una o varias partes de la cadena determinan el carácter total del encadenamiento, y son responsables del fomento de las actividades a través de acoplamientos individuales y su manejo. El esquema y proceso, así definido, es lo que se conoce como gobernanza (Kaplinsky y Morris, 2000: 8).

El término gobernanza acentúa el papel de liderazgo adquirido por una o varias firmas del encadenamiento, las cuales son las encargadas de organizar la cadena mediante reglas, parámetros, leyes $\mathrm{y} / \mathrm{o}$ formas comportamentales de los nodos. El tipo de gobernanza influye en los métodos de producción, los procesos de aprendizaje y en las estrategias de desarrollo local, a través de una estructura basada en relaciones y mecanismos institucionales que viabilizan la coordinación (Messner, 2002: 21).

El punto central es entender y precisar cómo se estructura la gobernanza existente en una CGV. Humphrey y Schmitz (2002:24) advierten tres tipos de interrogantes o parámetros, definidos por las firmas líderes: qué producir, cómo producir y el flujo físico del producto. La manera en que estas decisiones son estructuradas y tomadas, así como las actividades entre las firmas y su coordinación, describen el tipo de gobernanza prevaleciente (Messner, 2002: 22).

Con el uso del término gobernanza, es posible identificar el liderazgo ejercido por una o varias de las firmas, localizadas en los diferentes nodos, las cuales elaboran y monitorean los parámetros que el resto debe acatar. También se pone especial énfasis en la articulación de encadenamientos mercantiles globales con especialización flexible, cuyas etapas de producción, distribución y consumo están vinculadas por relaciones sociales $u$ organizacionales. Asimismo, a través de la noción de CGV, se promueve el análisis del espacio de estructuras económicas imperfectas, en términos de acceso diferencial a mercados y recursos.

La gobernanza facilita que las interacciones entre las firmas, respondan a una acción coordinada de organización y no a la mera casualidad. Ello revela que las cadenas se gobiernan, cuando los parámetros que requiere el producto, el proceso y la logística, están estrechamente vinculados a actividades, actores, roles y funciones de los nodos que la integran (Kaplinsky y Morris, 2000: 29).

De esta forma, el término gobernanza se utiliza para enfatizar el rol de liderazgo que ciertas empresas ejercen. Es posible afirmar que una CGV sin gobernanza es sólo una red de relaciones de mercado, al no existir una firma que marque las pautas a seguir en el encadenamiento. Su estudio permite entender lo siguiente: 1) las formas de acceso al mercado, donde las firmas líderes integran y coordinan actividades internacionalmente dispersas, ya que su toma de decisiones pueden conducir hacia afuera del encadenamiento a productores y comerciantes; 2) la rápida adquisición de las habilidades de producción, donde los productores tienden a encontrarse en una curva de aprendizaje, ante la exigencia de bajos costos, aumento de calidad y velocidad del servicio; 3) la distribución de la ganancia a lo largo de la cadena; 4) las iniciativas de política gubernamental que se proponen en la CGV no enfocadas a relaciones de 
mercado; 5) la asistencia técnica que se brinda a pequeños y medianos proveedores entrantes localizados en distancias lejanas de las firmas gobernantes (Humphrey y Schmitz, 2001: 2-3).

Los actores clave dentro de una CGV toman la responsabilidad de potenciar las capacidades delos participantes involucrados en una misma actividad, fragmentados entre las distintas firmas involucradas. En la cadena convergen diferentes agentes, quienes trabajan cercanos unos con otros $\mathrm{y}$ mantienen una intensa relación dentro $\mathrm{y}$ fuera del encadenamiento, dando origen a un sistema de producción social que eleva la motivación y la capacidad de la empresa o empresas para innovar procesos, servicios o productos.

Conforme se manifiesta la gobernanza, es posible distinguir dos diferentes tipos de $\mathrm{CGV}$ (ver Cuadro 1). El primero hace referencia a las cadenas donde el rol de la gobernanza

Cuadro 1. Características principales de las cadenas globales de valor conducidas por el comprador y el productor

\begin{tabular}{|l|l|l|}
\hline \multicolumn{1}{|c|}{ Caracteristicas } & $\begin{array}{l}\text { Cadenas Conducidas por el } \\
\text { Comprador (CCC) }\end{array}$ & \multicolumn{1}{|c|}{$\begin{array}{c}\text { Cadenas Conducidas por el } \\
\text { Productor (CCP) }\end{array}$} \\
\hline Conductores de las cadenas & Capital comercial & Capital industrial \\
\hline Puntos de competencia & Diseño, mercadeo. & Investigacióny desarrollo, producción. \\
\hline Barteras a la entrada & Economías de alcance & Economías de escala \\
\hline Sectores económicos & Bienes perecederos & $\begin{array}{l}\text { Bienes de consumo duraderos, bienes } \\
\text { intermediosy bienes de capital. }\end{array}$ \\
\hline Tipo de industria & Ropa, calzado y juguetes. & Automóvil, computadora, aérea. \\
\hline $\begin{array}{l}\text { Propiedad de la empresa } \\
\text { manufacturera }\end{array}$ & $\begin{array}{l}\text { Locales, fundamentalmente en } \\
\text { paises en desarrollo. }\end{array}$ & Transnacionales \\
\hline $\begin{array}{l}\text { Vinculos principales de la la } \\
\text { red }\end{array}$ & Basados en el comercio & Basados en la inversión \\
\hline $\begin{array}{l}\text { Estructura de la red } \\
\text { dominante }\end{array}$ & Horizontal & Vertical \\
\hline
\end{tabular}

Fuente: Gereffi (2001: 19).

es desempeñado por el comprador (BuyerdrivenChain); las denominadas cadenas conducidas por el comprador (CCC) son relacionadas a las industrias intensivas en trabajo. El segundo, muestra un escenario donde los productores son la pieza clave de la cadena (Producer-drivenChain). En general, las cadenas conducidas por el productor (CCP) se destacan por el uso de tecnologías de punta, y el papel relevante del productor en la coordinación de los diversos acoplamientos.

Ambos tipos de encadenamientos son útiles para analizar y evaluar las industrias globales, pero, no hacen mención de aquellos casos donde un actor intermedio ejerce la gobernanza en la cadena. Al estudiar la exportación hortícola sinaloense, se percibe la existencia de una cadena de valor, dirigida en sus inicios y en varios periodos de su historia, por agentes distribuidores localizados en un nodo intermedio. Como se anota en el siguiente capítulo, fueron los distribuidores los encargados de potenciar los cultivos de hortalizas en Sinaloa y pioneros en la formación de un puente comercial hacia EE.UU. Por lo anterior, es posible hablar de la existencia de Cadenas Conducidas por el Distribuidor (CCD).

En atención a las características de las cadenas, definidas en el Cuadro 1, las CCD se conforman por capital comercial, basan su competencia en la producción, pero sobre todo en la presentación y la calidad de las mercancías; se organizan en las actividades relacionadas con bienes perecederos $\mathrm{e}$ intermedios de la industria hortícola. Los propietarios de las empresas de exportación en las cadenas son residentes locales de un país o región en desarrollo, los cuales crean vínculos mediante el comercio para formar redes, a través de la integración vertical y horizontal.

Las CCD tienen su origen en economías donde la división de la actividad entre regiones no incluye un gran número de intermediarios en la distribución y venta del producto al consumidor final; además, existe una estrecha relación entre el distribuidor 
y el productor. Los distribuidores - como encargados de ejercer la gobernanzatienen un papel activo en la definición del tipo de mercancía a producir, en el financiamiento de la producción, y en la promoción y comercialización del producto. Estos encadenamientos existen, muy probablemente, en actividades económicas nacientes, necesitadas de participantes promotores y gestores.

Los estudios sobre las diferentes $\mathrm{CGV}$, indican que el comercio global está organizado en redes estables de corporaciones legales independientes unas de otras, donde el intercambio no es resultado de mercados anónimos, sino de la coordinación y secuencia de actividades (logística, empacado, mercado, entre otras). La gobernanza se presenta de formas diversas, tales como: baja gobernanza, puntos múltiples de gobernanza o combinación de varias, en acuerdo a los diversos tipos de cadenas (CCC, CCP y CCD). Asimismo, los encadenamientos se asientan sobre ventajas comparativas (factores de precio) y competitivas (factores distintos del precio) asociadas a la geografía, hecho que facilita la transferencia de tecnología y el acercamiento al mercado final.

Cada tipo de gobernanza es capaz de intervenir en el acceso a mercados globales, en el conocimiento y en la distribución de la ganancia entre empresas en una $\mathrm{CGV}$; también en los modelos de inversión y producción global, integración o exclusión de industrias locales al mercado global (Messner, 2002: 25). Las firmas líderes representan el punto de entrada al mercado internacional para una multiplicidad de empresas.

El hecho de que algunas cadenas son gobernadas por firmas líderes de países desarrollados influye significativamente en las empresas proveedoras, instaladas en países en desarrollo. Esta influencia ha sido reconocida por agencias gubernamentales y no gubernamentales, encargadas de establecer estándares globales (Messner, 2002), y ambientales y laborales, para el desempeño de una actividad en la economía global. Así, los responsables de la gobernanza pueden crear iniciativas en el ámbito laboral y ambiental, al tener un mejor conocimiento de los actores de un sector de la economía, así como por participar en iniciativas relacionadas con políticas comerciales, normas de calidad y sanidad.

\section{Distribución hortícola: su protagonismo}

La separación geográfica y en el tiempo, las formas de transportación entre compradores y vendedores, además de la imposibilidad de situar la base de producción hortícola cerca del consumidor, han hecho necesario el traslado de bienes y servicios desde su lugar de elaboración hasta el consumidor final a través de la distribución.

Por distribución se entiende la función que permite el traslado de productos y servicios desde la producción al de adquisición y consumo, abarcando un conjunto de actividades o flujos necesarios para situarlos a disposición del comprador (individuos $\mathrm{u}$ organizaciones) en las condiciones de lugar, tiempo, forma y cantidad adecuados.

Son diversas las funciones vinculadas a la distribución hortícola que demuestran el porqué esta fase no sólo trata el traslado de vegetales frescos a su destino final, sino que constituye una actividad compleja que incluye: compra y venta; transporte y difusión de la producción; fraccionamiento; almacenamiento; servicios vinculados $\mathrm{y}$ no vinculados directamente a la venta $\mathrm{y}$, 
financiamiento y asunción de riesgos.

Es posible diferenciar dos tipos de instituciones, relacionadas con la distribución: la primera, las instituciones principales, enfocadas a las actividades primordiales como compra y/o venta de productos, donde se localizan a productores, mayoristas, minoristas y consumidores finales; las segundas, las instituciones secundarias, con tareas de servicios que regulan y coordinan el proceso, a las que pertenecen empresas financieras, transporte, almacenistas independientes, compañías de seguro y publicidad.

Cuando un conjunto de participantes en el canal de distribución realizan de forma mancomunada todas o algunas de las funciones, se dice que existen estructuras coordinadas. Sus componentes actúan colectivamente para lograr mejores condiciones comerciales, asegurar sus ventas y conseguir economías de escala, con el fin de obtener una mejor posición y mayor poder en el mercado. Es posible diferenciar entre dos tipos de relaciones internas: coordinación y subordinación. La primera se basa en agrupaciones en el régimen de igualdad, en tanto la segunda implica que las empresas asociadas se someten a una dirección única, vertical u horizontal.

Los Sistemas Verticales de Marketing (SVM), estructuras coordinadas, reconocen y desean de sus integrantes la interdependencia. Las relaciones que existen en torno a la distribución hortícola son duraderas, siempre y cuando los resultados obtenidos de forma cooperativa superan los que se obtendrían de manera individual, sustentándose en la especialización, riesgo compartido, beneficios nivelados $\mathrm{y}$

El siguiente apartado recurre a Díez de Castro (2004) que hace una aplicación al caso de las hortalizas y su distribución, y que ha servido de fundamento y auxilio para la explicación del caso de estudio. creatividad compartida.

En otro sentido, los Sistemas Horizontales de Marketing (SHM) se caracterizan por agrupar a distribuidores del mismo nivel del canal. La finalidad es optimizar alguna de las funciones de la gestión de las empresas de distribución, para obtener una mejor posición o poder en el canal de venta, visualizada en la alta concentración de empresas.

Por lo general, los integrantes del canal de distribución efectúan sus transacciones de forma vertical, es decir, compran a proveedores situados en un nivel inferior y venden a componentes de uno superior. Pero también existe la posibilidad de que efectúan sus transacciones de forma horizontal, en el sentido de que una distribuidora compre y venda a otras, de forma mixta (horizontal y vertical), por ejemplo, una distribuidora que compra a otra y vende a mayoristas, minoristas o detallistas.

El tipo de control más generalizado en la distribución es el contractual, pues a través de éste se derivan los términos y condiciones que se incluyen en los contratos, definiendo las responsabilidades y recompensas de las partes en la realización de sus actividades.

La distribución tiene como objetivo que los productos estén disponibles para el comprador, y la obtención de relaciones estables, continuas y duraderas entre los demás miembros del canal de la comercialización, de forma que repercuta en una mayor satisfacción del cliente, facilitando y simplificando las operaciones, disminuyendo los costos, y logrando mayor eficacia y eficiencia. Es normal la existencia de una empresa líder que toma la dirección

Denominadas también paralelas, auxiliares o periféricas 
Cuadro 2. Clasificación de las Distribuidoras

\begin{tabular}{|ll|c|}
\hline \multicolumn{1}{|c|}{ Tipo } & Muestra \\
\hline - & Distribuidora mexicana que comercializa producto propio & 3 \\
- & Distribuidora mexicana que comercializa producto mixto & 9 \\
- Distribuidora estadounidense & 4 \\
\hline \multicolumn{2}{|l|}{} \\
\hline
\end{tabular}

Fuente. Elaboración propia.

y controla la totalidad, o parte del canal, dejando a las restantes con una relación de dependencia.

El concepto de poder utilizado en la literatura referida a la distribución, se describe como la existencia de una organización que supone la participación de otra u otras actuando o conllevando ser controladas, tolerancia fundamentada en el grado de dependencia que tienen las organizaciones y en la percepción de acceder a mayores utilidades o resultados bajo el control de la organización que tiene el poder.

La definición de poder que goza de mayor aceptación en los canales de distribución es la descrita como la habilidad de un miembro para controlar las variables decisionales en la estrategia de mercadeo de otro actor situado en un nivel distinto del canal. El poder consiste en la facilidad de influir o alterar las decisiones de otro(s).

Otra forma de ejercer poder es a través del liderazgo: la condición de superioridad en la que se haya una empresa dentro de su ámbito de actuación. El líder tiene capacidad para lograr la colaboración voluntaria del resto de participantes del canal, facilitando la cooperación interorganizacional y la coordinación de las diferentes actividades o funciones a realizar, lo cual permite controlar determinados aspectos de las operaciones que se llevan a cabo en el intercambio, con el propósito de conseguir los objetivos planteados; además, tiene la capacidad para resolver los problemas y gestionar los conflictos dentro del canal.

Por otra parte, el liderazgo puede ser asumido por cualquiera de los participantes. Además, una misma empresa puede ser líder en unas determinadas acciones y tolerar ser dominada en otras. Las relaciones son un proceso de regateo, cuyo objetivo es económico: precios, márgenes, comisiones, descuentos, ayudas publicitarias, costos de transporte y formas de pago, para conseguir los máximos resultados posibles.

La repartición de los beneficios depende de la posición y poder, de manera que el segundo permite realizar o modificar acciones individuales hacia comportamientos más coordinados.

El poder, liderazgo y control que ejercen las distribuidoras son estructuras de gobernanza que han practicado a lo largo de la historia de la CHVSEU; además, las firmas de distribución han tenido que adecuarse a los cambios de la economía global yserpartícipes de los estándares globales homogenizadores del comercio hortícola de EE.UU., con el resto del mundo. Ello ha permitido la integración de la producción sinaloense a un país con necesidad de abastecerse de vegetales frescos y convertirse en precursor de la actividad y en un nicho de producción a bajos costos. 
Índice de poder, liderazgo y control: ¿Quién manda?

Además de la revisión bibliográfica y hemerográfica, fue necesaria la realización de una investigación de campo en el área de Nogales, Arizona, donde se entrevistó a gerentes, subgerentes y encargados de venta de 16 empresas distribuidoras, además de horticultores en Sinaloa.

La muestra es diversa, del total de empresas entrevistadas cuatro son de capital estadounidense y el resto mexicano. Las distribuidoras norteamericanas sólo mercadean producto ajeno, es decir no tienen ninguna relación de parentesco o sociedad con los productores de origen. Por otro lado, tres de las compañías de capital mexicano se dedican exclusivamente a la comercialización de hortalizas de productos propios. El resto de las empresas mexicanas enfocan sus esfuerzos de venta a vegetales provenientes de productores propios y de otros (producto mixto) que no cuentan con una distribuidora de su propiedad en la frontera. Así, la clasificación de las distribuidoras se resume en el cuadro 2 .

El parentesco de los distribuidores con sus socios horticultores está relacionado con la pertenencia de un campo propio. Así, diez de las empresas entrevistadas tienen una relación de parentesco con sus productores y mencionan la existencia de un campo propio localizado en Sinaloa o Sonora. Ello denota un proceso de integración vertical de actividades.

Conforme a la clasificación de las distribuidoras, el productor que opera su propia distribuidora goza de ventaja competitiva en el mercado, lo cual se refleja

\footnotetext{
La selección del muestreo se tomó de un total de 42 distribuidoras, registradas en la FPAA, localizadas en Nogales, Arizona, las cuales fueron contactadas por correo electrónico y mediante llamadas telefónicas, el resultado fue la obtención de 16 entrevistas atendidas.
}

en la gama de clientes, donde destacan las grandes cadenas de supermercados: WalMart, Costco, Safeway, Kroger, entre otras. La distribuidora, mediante su dinámica, trata de apoderarse del mayor margen de utilidad posible al vender hortalizas de otros productores. Si proporciona financiamiento, procura concretar alguna ganancia contractual.

Los datos recopilados en la investigación de campo fueron utilizados para la construcción de un índice de poder, liderazgo y control, mediante el cual fue posible detectar, de acuerdo con la clasificación de las distribuidoras cuáles son los actores más importantes en la comercialización hortícola.

Las variables de poder, liderazgo y control son centrales en la formación del concepto gobernanza. Para identificarlas y medirlas, en un primer momento, se realiza una revisión de las diversas definiciones y clasificaciones; posteriormente se transcribe la información en preguntas, y a partir de los resultados se establece una fórmula que permite medir su incidencia en cada actor de la comercialización hortícola en el mercado estadounidense: productores, distribuidores, centrales de abasto (mayoristas) y cadenas de supermercados.

El índice tiene la siguiente limitante, es una muestra de la opinión de los distribuidores y no incluye las consideraciones de los demás participantes del encadenamiento. El interés se centró en saber cómo se aprecia, desde la perspectiva del distribuidor, la gobernanza en la cadena, y así entender su relación con el resto de los actores de la exportación hortícola. 
La fórmula general para medir el poder, liderazgo y control se elabora a partir del número de menciones de los actores, como respuesta, en cada una de las preguntas. Los cuestionamientos se tomaron como factores, siendo los siguientes:

Factor 1 (F1): ¿Qué actor o participante de la cadena de comercialización hortícola piensa tiene el papel más importante en la exportación hortícola?

Factor 2 (F2): ¿Qué participante de la cadena productiva impone la forma en que se lleva a cabo la comercialización de exportación?

Factor 3 (F3): ¿Quién indica las modificaciones o cambios necesarios en las etapas de producción, exportación, distribución y comercialización hortícolas?

Una vez hecha la suma de las menciones de un actor-por ejemplo, un productorpara cada factor, uno a uno se dividen por el número de empresas del muestreo, se hace el cálculo por tipo de distribuidora, y se multiplica por la fracción cuyo nominador es uno. El denominador son las variables de los factores, las cuales corresponden al número de opciones de respuesta de cada pregunta: productor, distribuidor, cadena de supermercado y central de abasto. Una vez multiplicadas las fracciones para cada uno de los factores, se suman los resultados de los tres y el total se divide de nuevo entre el número total de factores que también corresponde a tres. La fórmula se muestra a continuación:

$$
I P_{i}=\frac{\frac{M i_{F 1}}{E M}\left(\frac{1}{V_{F 1}}\right)+\frac{M i_{F 2}}{E M}\left(\frac{1}{V_{F 2}}\right)+\frac{M i_{F 3}}{E M}\left(\frac{1}{V_{F 3}}\right)}{N F}
$$

Donde:

$I P_{i}=$ Indicador de poder, liderazgo y control del actor $\mathrm{i}$
$M i_{F i}=$ Menciones del actor en el factor $\mathrm{i}$

$E M=$ Número de empresas del muestreo

$V_{F i}=$ Variables del factor $\mathrm{i}$

$N F=$ Número total de factores

La fórmula sintetizada es:

$$
I P_{i}=\frac{\sum_{i=1}^{n} \frac{M i_{F i}}{E M}\left(\frac{1}{V_{F i}}\right)}{N F}
$$

El número resultante de los cálculos de los actores de la cadena muestra cuál ejerce mayor poder, liderazgo y control en la CHVSEU. El rango de valores es de 0 a 0.25; es decir, el valor mínimo que puede obtener un actor en el índice es de cero, al no tener ninguna mención, y el máximo es de 0.25 , al tener todas las menciones. A continuación se presentan la fórmula por actor y el resultado numérico.

Fórmula para productores

$$
\begin{gathered}
I P_{P}=\frac{\sum_{i=1}^{n} \frac{M P_{F i}}{E M}\left(\frac{1}{V_{F i}}\right)}{N F} \\
I P_{P}=\frac{\frac{M P_{F 1}}{E M}\left(\frac{1}{V_{F 1}}\right)+\frac{M P_{F 2}}{E M}\left(\frac{1}{V_{F 2}}\right)+\frac{M P_{F 3}}{E M}\left(\frac{1}{V_{F 3}}\right)}{N F}
\end{gathered}
$$

Con cifras por tipo de distribuidora

Distribuidoras mexicanas que comercializan producto propio

$$
I P_{P}=\frac{\frac{3}{3}\left(\frac{1}{4}\right)+\frac{0}{3}\left(\frac{1}{4}\right)+\frac{1}{3}\left(\frac{1}{4}\right)}{3}=\frac{0.25+0.083}{3}=0.1111
$$


Distribuidoras mexicanas que comercializan producto mixto

$$
I P_{P}=\frac{\frac{5}{9}\left(\frac{1}{4}\right)+\frac{0}{9}\left(\frac{1}{4}\right)+\frac{3}{9}\left(\frac{1}{4}\right)}{3}=\frac{0.138+0.083}{3}=0.0737
$$

Distribuidoras estadounidenses

$$
I P_{P}=\frac{\frac{3}{4}\left(\frac{1}{4}\right)+\frac{0}{4}\left(\frac{1}{4}\right)+\frac{0}{4}\left(\frac{1}{4}\right)}{3}=\frac{0.1875}{3}=0.0625
$$

Fórmula para distribuidoras

$$
\begin{aligned}
I P_{D} & =\frac{\sum_{i=1}^{n} \frac{M D_{F i}}{E M}\left(\frac{1}{V_{F i}}\right)}{N F} \\
I P_{D} & =\frac{\frac{M D_{F 1}}{E M}\left(\frac{1}{V_{F 1}}\right)+\frac{M D_{F 2}}{E M}\left(\frac{1}{V_{F 2}}\right)+\frac{M D_{F 3}}{E M}\left(\frac{1}{V_{F 3}}\right)}{N F}
\end{aligned}
$$

Distribuidoras mexicanas que comercializan producto mixto

$$
I P_{C S}=\frac{\frac{0}{3}\left(\frac{1}{4}\right)+\frac{0}{3}\left(\frac{1}{4}\right)+\frac{0}{3}\left(\frac{1}{4}\right)}{3}=0
$$

Distribuidoras mexicanas que comercializan producto propio

Con cifras por tipo de distribuidora

$$
I P_{C S}=\frac{\frac{3}{9}\left(\frac{1}{4}\right)+\frac{6}{9}\left(\frac{1}{4}\right)+\frac{5}{9}\left(\frac{1}{4}\right)}{3}=\frac{0.0833+0.1666+0.1388}{3}=0.1295
$$

Distribuidoras estadounidenses

Distribuidoras mexicanas que comercializan producto propio

$$
I P_{C S}=\frac{\frac{1}{4}\left(\frac{1}{4}\right)+\frac{2}{4}\left(\frac{1}{4}\right)+\frac{1}{4}\left(\frac{1}{4}\right)}{3}=\frac{0.0625+0.125+0.0625}{3}=0.0833
$$

$$
I P_{D}=\frac{\frac{1}{3}\left(\frac{1}{4}\right)+\frac{0}{3}\left(\frac{1}{4}\right)+\frac{0}{3}\left(\frac{1}{4}\right)}{3}=\frac{0.833}{3}=0.0277
$$

Distribuidoras mexicanas que comercializan producto mixto

$$
I P_{D}=\frac{\frac{4}{9}\left(\frac{1}{4}\right)+\frac{1}{9}\left(\frac{1}{4}\right)+\frac{2}{9}\left(\frac{1}{4}\right)}{3}=\frac{0.111+0.027+0.055}{3}=0.0643 I P_{C A}=\frac{\frac{M C A_{F 1}}{E M}\left(\frac{1}{V_{F 1}}\right)+\frac{M C A_{F 2}}{E M}\left(\frac{1}{V_{F 2}}\right)+\frac{M C A_{F 3}}{E M}\left(\frac{1}{V_{F 3}}\right)}{N F}
$$

Distribuidoras estadounidenses

$$
I P_{D}=\frac{\frac{1}{4}\left(\frac{1}{4}\right)+\frac{0}{4}\left(\frac{1}{4}\right)+\frac{0}{4}\left(\frac{1}{4}\right)}{3}=\frac{0.0625}{3}=0.0208
$$

Fórmula para Centrales de Abasto

$I P_{C A}=\frac{\sum_{i=1}^{n} \frac{M C A_{F i}}{E M}\left(\frac{1}{V_{F i}}\right)}{N F}$

Con cifras por tipo de distribuidora

Distribuidoras mexicanas que comercializan producto propio

$$
I P_{C A}=\frac{\frac{0}{3}\left(\frac{1}{4}\right)+\frac{3}{3}\left(\frac{1}{4}\right)+\frac{2}{3}\left(\frac{1}{4}\right)}{3}=\frac{0.25+0.16}{3}=0.1388
$$


Distribuidoras mexicanas que comercializan producto mixto

$$
I P_{C A}=\frac{\frac{1}{9}\left(\frac{1}{4}\right)+\frac{1}{9}\left(\frac{1}{4}\right)+\frac{2}{9}\left(\frac{1}{4}\right)}{3}=\frac{0.027+0.027+0.0555}{3}=0.0365
$$

Distribuidoras estadounidenses

$$
I P_{C A}=\frac{\frac{2}{4}\left(\frac{1}{4}\right)+\frac{2}{4}\left(\frac{1}{4}\right)+\frac{2}{4}\left(\frac{1}{4}\right)}{3}=\frac{0.125+0.125+0.125}{3}=0.125
$$

en constante competencia, por la mayor penetración en el mercado estadounidense, asegura el cumplimiento de las solicitudes impuestas.

Las cifras indican que el distribuidor se visualiza en una posición intermedia, entre los productores y clientes, con poco rango de negociación, al no ubicarse como el actor principal, en ninguno de los casos, en el

\section{Cuadro 3. Resultados del índice de poder, liderazgo y control}

\begin{tabular}{|l|l|l|l|l|}
\cline { 2 - 5 } \multicolumn{1}{c|}{} & \multicolumn{4}{c|}{ Actor } \\
\hline Tipo de distribuidora & Productor & Distribuidor & $\begin{array}{c}\text { Cadena de } \\
\text { supermercado }\end{array}$ & $\begin{array}{c}\text { Centrales de } \\
\text { abasto }\end{array}$ \\
\hline $\begin{array}{l}\text { Distribuidoras mexicanas } \\
\text { que comercializan } \\
\text { producto propio }\end{array}$ & 0.1111 & 0.0277 & 0 & 0.1388 \\
\hline $\begin{array}{l}\text { Distribuidoras mexicanas } \\
\text { que comercializan } \\
\text { producto mixto }\end{array}$ & 0.0737 & 0.0643 & 0.1295 & 0.0363 \\
\hline $\begin{array}{l}\text { Distribuidoras } \\
\text { estadounidenses }\end{array}$ & 0.0625 & 0.0208 & 0.0833 & 0.125 \\
\hline
\end{tabular}

Fuente: Elaboración propia

En resumen, los resultados por actor de la cadena y tipo de distribuidora son:

En el caso de las empresas que comercializan producto propio, los resultados indican que las cadenas de supermercados no son su principal cliente; ese rol lo desempeñan los mayoristas, como las centrales de abasto. Lo mismo sucede en el caso de las empresas norteamericanas.

Para las distribuidoras que comercializan producto mixto, las cadenas de supermercado son el actor más importante en el encadenamiento y sus principales clientes. Estas cadenas prefieren relacionarse con empresas mexicanas que les garanticen producto de calidad, en los volúmenes y presentaciones solicitados; además, el hecho de que los distribuidores mexicanos estén ejercicio de poder, liderazgo y control de la cadena. Por el contrario, los productores expresan que las distribuidoras tienen total libertad en la negociación de sus productos.

\section{CONCLUSIONES}

La gobernanza es como un campo de disputa. La cadena se construye y articula mediante la participación y coordinación de los agentes. En su origen, antes de la globalización y la diversidad de competidores, el productor simplemente quería llegar al mercado y obtener un porcentaje de las ventas, la competencia era local. Al evolucionar la cadena y aumentar la competencia, las posibilidades de negocio se elevan, aparecen nuevas alternativas, nuevas reglas y mayores exigencias, impulsando a los diferentes actores económicos a compartir 
segmentos de la gobernanza de las cadenas globales de valor.

En este esquema, la distribución es un eslabón importante en el encadenamiento de las actividades entre países o regiones, en particular para la formación de la cadena hortícola de valor entre Sinaloa y Estados Unidos. La distribuidora en su papel de comprador centralizado organiza el comercio de hortalizas en EE.UU., operando para productores, brokers, mayoristas, minoristas e institucionales, que con el paso del tiempo han ido alterando su rango de acción.

En los inicios de la horticultura de exportación sinaloense, existía una relación cercana con los productores al tener oficinas próximas a los campos de producción. Tiempo después, las modificaciones en la estructura de mercado, el incremento de la población y de empresas ofertantes de vegetales frescos, obligan su localización en un punto intermedio del comprador y el productor, provocando una aglomeración de distribuidoras en algunos puntos fronterizos como Nogales, Sonora-Arizona. De esa forma, los objetivos de las distribuidoras se traducen en coordinar y controlar el proceso de exportación, liderar las ventas en el mercado y ejercer poder de decisión en el proceso de producción, en cuanto al tipo del producto, su calidad y cantidad, acciones que forman parte de la gobernanza que ejercen en la CHVSEU.

Con el paso del tiempo y las nuevas exigencias las distribuidoras han debido innovar en su forma de comercializar con la adquisición de nuevos tipos de servicios basados en investigaciones de oferta $y$ demanda, y en la elaboración de manuales de calidad. También buscan ampliar su perspectiva de desarrollo y su penetración en los mercados de los productos que se ofrecen.
No es para menos, su responsabilidad radica en la vinculación del horticultor con los diferentes canales de venta, en su influencia en las transacciones que se derivan de llevar el producto, desde el campo al mercado terminal, y en las primeras estimaciones del precio. Esto último basado en la demanda del mercado, permitiendo evaluar los mejores periodos de venta, las cantidades a colocar y fechas de plantación.

Así, una de las labores primordiales de la distribuidora es tener prestigio entre los clientes que atiende $\mathrm{y}$, de esta forma, incrementar su cantidad; por ello la calidad de sus productos, su presentación y el que sean enviados en los tiempos establecidos es determinante en su desempeño. Ante esta preocupación, algunos productores sinaloenses, a través de la integración vertical, detectaron la posibilidad de controlar desde el punto de origen hasta el de venta la movilidad de sus productos, estableciendo distribuidoras propias.

Sin importar la procedencia del capital de la empresa de distribución tienen presente que las modificaciones del mercado han colocado a los supermercados como los actores más importantes en la venta al detalle, repercutiendo en el esquema de comercialización de hortalizas y en la gobernanza en la CHVSEU, que atiende una jerarquía que va del supermercado a la distribuidora, y de la distribuidora al productor.

Ello no quiere decir que el resto de los actores de la cadena hayan perdido presencia, en especial para distribuidoras de capital mexicano que comercializan producto propio y las estadounidenses, que tienen como cliente principal a las centrales de abasto. Tampoco indica que las distribuidoras estén limitadas en cuanto a los 
clientes que abastecen, al contrario tienen la facilidad y posibilidad de atender cualquier miembro de mercado.

El hecho de que los supermercados tengan una relación directa con el consumidor y el distribuidor ha impactado en un incremento de la calidad, exigencia que ha repercutido en la flexibilidad de los productores para satisfacerla, abriendo nuevos nichos de crecimiento y oportunidad de mayor penetración de los productos hortícolas sinaloenses, confirmando, de tal forma, el necesario ejercicio de poder, liderazgo y control que realizan las distribuidoras en el encadenamiento.

\section{LITERATURA CITADA}

Avilés Ochoa, Ezequiel. 2006. Crecimiento y Política Económica. Sinaloa frente a México. Universidad de Occidente, Doctorado en Ciencias Sociales de la Universidad Autónoma de Sinaloa y Dirección de Investigación y Fomento a la Cultura (DIFOCUR)

Carton de Grammont, Hubert 1990. Los empresarios agrícolas y el estado. Sinaloa, 1893-1984, Universidad Nacional Autónoma de México, Instituto de Investigaciones Sociales, México, DF.

Carton de Grammont, Hubert (coord.). 1999. Empresas, reestructuración productiva y empleo en la agricultura mexicana, Instituto de Investigaciones Sociales, UNAM, Plaza y Valdés, México.

Carton de Grammont, Hubert. 2006. Las empresas, el empleo y la productividad del trabajo en la horticultura de exportación, en prensa, Instituto de Investigaciones Sociales, México, DF.
Cook, Roberta. 2003a. The envolving global market place for fruit and vegetables, Agricultural Issues Center and Department of Agricultural and Resource Economics, University of California, Davis, Abril, EE UU.

Díez de Castro, Enrique. 2004. Distribución comercial, tercera edición, Mc Graw Hill, España.

Flores García, Silvia Raquel. 1987. Nogales, un siglo de historia, INAH-SEP, Centro Regional del Noroeste, Secretaría del Fomento Educativo y Cultura, Hermosillo, Sonora, México.

Fujita, Masahisa, Paul Krugman y Anthony J. Venables. 2000. Economía espacial, las ciudades, las regiones y el comercio internacional, Ed. Ariel Economía, España.

Gereffi, Gary y Miguel Korzeniewicz. 1994. Commodity chains and global capitalism, PraegerPublichers, EE UU.

Gereffi, Gary. 2001. "Las cadenas productivas como marco analítico para la globalización" en Problemas del Desarrollo, Vol. 32, Núm. 125, abril-junio, IIEC-UNAM, México.

Gereffi, Gary, John Humphrey y Timothy Sturgeon. 2003. "The governance of global value chains" [en línea]. Global value chain initiative. $<$ http:// www.ids.ac.uk/globalvaluechains/ publications/govgvesfinal.pdf $>$ [Consulta: enero 2005]

Gereffi, Gary. 2005. "The global economy: organization, governance, and development" en Handbook 2005.

González, Humberto y Margarita Calleja. 1998. La exportación de frutas y hortalizas a Estados Unidos de Norteamérica, Ed. SAGAR, CIESAS 
y CONACYT, México, DF.

González, Humberto y Margarita Calleja. 1999. "Agricultura de exportación en tiempos de globalización", en Schwentesius, Rita, Manuel Ángel Gómez, Humberto González y Hubert C. de Grammont. Agricultura de exportación en tiempos de globalización. El caso de las hortalizas, frutas y flores, CIESTAAM-UACH, IIS-UNAM, CIESAS, Juan Pablos Editor, S.A., México, DF.

Humphrey, John, y Huber Schmitz. 2001. "Governance in global value chains" en IDS Bulletin, Vol 32, No 3, Industrial Performance Center, Massachusetts Institute of Technology.

Kaplinsky, Raphael y Mike Morris. 2000. “A Handbook for value chain research" [en línea].[Gran Bretaña], Institute of development studies.<http:// www.ids.ac.uk/ids/global/pdfs/ vchnov01.pdf\#search=' A \%20 $\mathrm{H}$ A N D B O O K \% 20 F O R \% 20 VALUE\%20CHAIN'> [Consulta: enero 2005]

López Barraza, Lydia Ma. 2004. La Estructura comercial de las hortalizas mexicanas en el suroeste de Estados Unidos: frontera con Nogales, (tesis de maestría) Maestría en Estudios de Estados Unidos y Canadá, UAS, Culiacán, Sin., septiembre.

Málaga, Jaime. 2000. “Tendencias recientes en el rol de los supermercados en la comercialización de frutas $y$ hortalizas en los Estados Unidos" en Schwentesius.

Rita y Manuel Ángel Gómez. 2000. Internacionalización de la horticultura, Universidad Autónoma de Chapingo, CIESTAAM, Ediciones Mundi-Prensa, México, DF, pp. 169-186.

Mares, David R. 1991. La irrupción del mercado internacional en México. Consideraciones teóricas $\mathrm{y}$ un estudio de caso, El Colegio de México, México.

Messner, Dirk. 2002. "The concept of the 'world economic triangle': global governance patterns and options for regions" [en línea].Global valuechaininitiative. $<$ http://www. ids.ac.uk/ids/bookshop/wp/wp173. pdf $>$ [Consulta: enero 2005]

Porter, Michael. 1980. Competitive Strategy, Free Press, New York, EE. UU.

Sinagawa Montoya, Herberto. 1987. Sinaloa, agricultura y desarrollo, CAADES, Culiacán, Sinaloa, México.

VanSickle, John J. ,et al. (1994). Competition in the U.S. winter fresh vegetable industry, Agricultural Economic Report Number 691, Economic Research Service, USDA, Washington, DC, EE. UU.

Ezequiel Avilés-Ochoa, Economista con Maestría en Economía por el Instituto Tecnológico Autónomo de México, Doctorado en Ciencias Sociales y Posdoctorado en Desarrollo Regional por la Universidad Autónoma de Sinaloa. Profesor Investigador de la Universidad de Occidente. Pertenece al Sistema Nacional de Investigadores Nivel II. Autor de varias obras en el campo de la economía y las finanzas. Ponente en congresos internacionales de investigación sobre la medición del progreso y bienestar social. Correo electrónico: eaviles@culiacan.udo. $\mathrm{mx}$. 
PODER, LIDERAZGO Y CONTROL DE LAS DISTRIBUIDORAS EN LA HORTICULTU-

RA DE EXPORTACIÓN SINALOENSE

Lydia María López-Barraza, Economista con Doctorado en Ciencias Sociales por la Universidad Autónoma de Sinaloa. Coordinadora de la Maestría en Administración de la Universidad de Occidente, Unidad Culiacán. Pertenece al Sistema Sinaloense de Investigadores y Tecnólogos del Consejo Estatal de Ciencia y Tecnología del Estado de Sinaloa. Ha participado en varias obras y congresos enfocados a la agricultura de exportación. Correo electrónico: lbarraza@culiacan.udo.mx. 